\title{
Shear-induced displacement of isotropic-nematic spinodals
}

\author{
T. A. J. Lenstra \\ Debye Institute, Van't Hoff laboratory, Utrecht University, Padualaan 8, 3584 CH Utrecht, The Netherlands
}

Z. Dogic

Complex Fluids Group, Physics Department, Brandeis University, Waltham, Massachusetts 02454

J. K. G. Dhont

Forschungszentrum Jülich, Institut für Festkörperforschung, Weiche Materie, 52425 Jülich, Germany

(Received 13 November 2000; accepted 28 March 2001)

\begin{abstract}
The shear dependent location of the isotropic-nematic spinodals in suspensions of bacteriophage fd is studied by means of time resolved birefringence experiments. The hysteresis in the birefringence on increasing and subsequently decreasing the shear-rate allows the determination of the location of points in the shear-rate vs concentration phase diagram between the isotropic-to-nematic and the nematic-to-isotropic spinodals. Experimental hysteresis curves are interpreted on the basis of an equation of motion for the orientational order parameter tensor, as derived from the N-particle Smoluchowski equation. The spinodals are found to shift to lower concentrations on increasing the shear-rate. Above a critical shear-rate, where shear forces dominate over thermodynamic forces, no spinodal instability could be detected. (C) 2001 American Institute of Physics.
\end{abstract}

[DOI: $10.1063 / 1.1372510]$

\section{INTRODUCTION}

Early experimental observations of isotropic-nematic phase coexistence in systems of rodlike colloidal particles were made by Zocher ${ }^{1}$ and Bernal. $^{2}$ In later experimental studies, ${ }^{3-8}$ other colloidal systems were found to exhibit isotropic-nematic phase coexistence as well, and in some studies $^{9-13}$ the kinetics of isotropic-to-nematic phase separation has been addressed. The isotropic-nematic phase transition has also been studied in an externally applied magnetic field. ${ }^{14}$ An exact theoretical result for the isotropic-nematic phase boundaries for very long and thin, rigid rods with excluded volume interactions was obtained some 50 years ago by Onsager. ${ }^{15}$ More recent theoretical developments on the isotropic-nematic phase transition can be found in Refs. 1618. Orientational order parameter dynamics in systems of rodlike colloids with excluded volume interactions have been considered theoretically by Doi and Edwards. ${ }^{19}$ Their approach is extended by Shimada et al. ${ }^{20}$ and very recently by Winters et al. ${ }^{21}$ to include inhomogeneities, in order to describe phase separation kinetics during the initial stage of spinodal demixing. Issues still open in this area are concerned with the role of attractive interactions and phase separation kinetics, especially beyond the initial stage (see van der Schoot $^{18}$ and Winters ${ }^{21}$ ).

Phase coexistence and phase separation kinetics in systems of lyotropic rigid rods under shear flow are less well understood. For wormlike micelles, experiments on phase separation and coexistence under shear flow are described in Ref. 22.

Shear flow tends to align rod-shaped colloids along the fluid flow direction. Rod-shaped particles that would constitute an isotropic system in the absence of shear flow will slightly align in the presence of shear flow. Such a shearaligned system is referred to as "paranematic" instead of "isotropic." At sufficiently high concentration, coexistence under shear flow conditions between a paranematic and a nematic phase may occur. The nematic phase has a higher degree of alignment than the paranematic phase. Of course, shear flow (when sufficiently weak) increases the degree of alignment of the nematic phase as compared to its unsheared state.

The only work on rodlike systems in this respect of which we are aware is due to Olmsted and co-workers. ${ }^{23-25}$ This work is partly based on thermodynamic considerations, where driving forces are assumed to be generated by gradients in free energy. Olmsted et al. assume local equilibrium and one of the phase coexistence conditions is equality of chemical potential in both phases. Although strictly speaking a free energy and chemical potential cannot be defined for a system in a nonconservative external field like the simple shear flow, this work predicts interesting features. Olmsted addresses the shear-induced shift of spinodals and binodals, the existence of a log-rolling phase, where the director of the aligned rods is parallel to the vorticity direction, and the occurrence of a hydrodynamic instability commonly referred to as the shear-banding instability. As far as we know, there are no experimental results on the shear-induced shift of phase coexistence boundaries or spinodals. In the present paper we present experimental results on the shear-induced shift of the isotropic-nematic spinodals for suspensions of bacteriophage $\mathrm{fd}$. The experimental technique that we used is time resolved birefringence measurement, where the hysteresis in birefringence on increasing and subsequently decreasing the shear-rate is measured.

This paper is organized as follows. In Sec. II B we shall present the theoretical background that is necessary to interpret experiments. In particular, we shall define the notion of spinodals in systems under shear flow, derive an equation of 


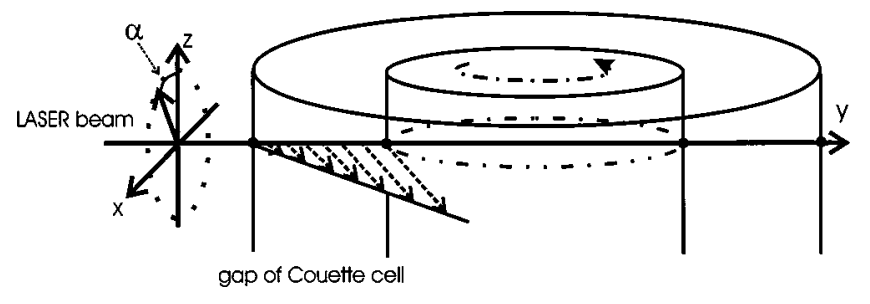

FIG. 1. Definition of the flow geometry.

motion for the orientational order parameter tensor, starting from the N-particle Smoluchowski equation for rigid rods, and use this equation of motion to calculate hysteresis curves. In the experimental Sec. III, we shall first describe the colloidal system and the birefringence equipment, and then proceed with presenting the experimental results and discussion. Section IV contains a summary and conclusions.

\section{THEORY}

\section{A. The suspension flow field}

The flow considered here is chosen in the $x$-direction, with its gradient in the $y$-direction. That is, the flow velocity is equal to $\mathbf{U}=\boldsymbol{\Gamma} \cdot \mathbf{r}$, where $\boldsymbol{\Gamma}$ is the velocity gradient tensor,

$$
\boldsymbol{\Gamma}=\dot{\gamma}\left(\begin{array}{lll}
0 & 1 & 0 \\
0 & 0 & 0 \\
0 & 0 & 0
\end{array}\right)
$$

with $\dot{\gamma}$ the shear-rate. The direction of the incident laser beam is along the gradient direction, as depicted in Fig. 1, while the polarization state of the light is characterized by the angle $\alpha$ of the electric field with the $z$-axis.

\section{B. Definition of spinodals in systems under shear flow}

A spinodal is defined as the set of control variables (like temperature, concentration, and shear-rate) where the system in the homogeneous state becomes absolutely unstable. That is, the spinodal separates the (meta-) stable region from the unstable region in the phase diagram, where demixing is initiated by order parameter fluctuations of arbitrary small amplitude. On the (meta-) stable side of the spinodal, phase separation is induced only by fluctuations with an amplitude that exceeds some minimum, finite amplitude. Without shear flow, the spinodal can be obtained from thermodynamic considerations: by definition the spinodal is the set of variables where the free energy barrier for phase separation becomes equal to zero. Such a thermodynamic approach is no longer feasible for systems in a nonconservative external field, like a simple shear flow. The spinodal must now be obtained from equations of motion for the relevant order parameter. By definition, the location of the spinodal can now be obtained by a linear stability analysis of the equation of motion. The set of control variables where the equation of motion becomes absolutely unstable defines the spinodal. This kinetic definition of the spinodal should reduce to the usual thermodynamic definition in the absence of shear flow. In case of the gas-liquid transition for attractive spherical col- loids, the relevant order parameter is the density. The equation of motion for the density, as derived from the Smoluchowski equation for spheres, is indeed shown in Ref. 26 to reproduce the gas-liquid spinodal as obtained from thermodynamic considerations, when shear-rate contributions to the equation of motion are neglected. For the rodlike systems under consideration here, the equation of motion for the orientational order parameter becomes unstable beyond some concentration. This equation of motion will be derived in the next subsection.

Experimentally, the location of a spinodal cannot in principle, be detected to within arbitrary accuracy. Close to the theoretical spinodal, on the (meta-) stable side, the amplitude of order parameter fluctuations necessary to initiate demixing is small, but nonzero. When the change in free energy on creating an order parameter variation of such small amplitude is less than about $k_{B} T$, demixing will occur without any time delay, as if one quenched into the unstable region of the phase diagram. There is thus a gradual change of nucleation dominated demixing to spinodal demixing. This prevents a sharp experimental determination of the location of the spinodal.

\section{An equation of motion for the orientational order parameter tensor}

The reason for deriving an equation of motion for the orientational order parameter is twofold. First of all, a linear stability analysis of such an equation renders the shear-rate dependent location of the spinodals. Second, this equation of motion will be used in Sec. II F to analyze the experimental procedure used here to detect the location of the spinodals.

The equation of motion we will derive here from the $\mathrm{N}$-particle Smoluchowski equation for rigid rods is very similar to that obtained from the Doi-Edwards equation. ${ }^{19}$ The assumptions involved in the derivation of the equation of motion for the orientational order parameter tensor are most clearly revealed by starting from the N-particle Smoluchowski equation. We shall therefore discuss the derivation of this equation of motion, starting from the $\mathrm{N}$-particle Smoluchowski equation, in some detail below.

The central quantity is the orientational order parameter tensor, which is defined as

$$
\mathbf{S} \equiv\langle\hat{\mathbf{u}} \mathbf{u}\rangle,
$$

where $\hat{\mathbf{u}}$ is the unit vector along the long axis of a uniaxial rod, the "orientation" of the rod, and the brackets denote ensemble averaging. The eigenvector of this tensor with the largest eigenvalue is commonly referred to as "the director," and points into the preferred orientation of the rods. Equations of motion for $\mathbf{S}$ have been considered before by Hess, ${ }^{27}$ and Doi and Edwards. ${ }^{19}$ Hess's derivation is based on thermodynamic arguments, while Doi and Edwards start from their equation of motion for the probability density function (pdf) of the orientations of a rod. Here we will start from the N-particle Smoluchowski equation for the pdf $P$ of the positions $\left(\mathbf{r}_{1}, \mathbf{r}_{2}, \ldots, \mathbf{r}_{N}\right)$ and orientations $\left(\hat{\mathbf{u}}_{1}, \hat{\mathbf{u}}_{2}, \ldots, \hat{\mathbf{u}}_{N}\right)$ of all $\mathrm{N}$ rods in the system under consideration. In analyzing this equation of motion we will recover the equation of mo- 
tion used by Doi and Edwards, which is known to reproduce the Euler-Lagrange equation that complies with Onsager's free energy functional without shear flow. ${ }^{28}$ The Smoluchowski equation is a continuity equation for translational and rotational motion and reads

$$
\frac{\partial P}{\partial t}=-\sum_{j=1}^{N}\left[\nabla_{j} \cdot\left(\mathbf{v}_{j} P\right)+\hat{\mathcal{R}}_{j} \cdot\left(\boldsymbol{\Omega}_{j} P\right)\right],
$$

with $\mathbf{v}_{j}$ the translational velocity of $\operatorname{rod} j$ and $\boldsymbol{\Omega}_{j}$ its angular velocity, $\nabla_{j}$ is the gradient operator with respect to $r_{j}$, and $\hat{\mathcal{R}}_{j}$ is the rotation operator which is defined as

$$
\hat{\mathcal{R}}_{j}(\ldots)=\hat{\mathbf{u}}_{j} \times \nabla_{u_{j}}(\ldots),
$$

with $\nabla_{\mathbf{u}_{j}}$ the gradient operator with respect to the orientation $\hat{\mathbf{u}}_{j}$ of $\operatorname{rod} j$.

On the Brownian time scale, where there is a force and torque balance, the translational and rotational velocities of the rods can be expressed as functions of positions and orientations. To this end, a rod is represented as a string of spherical beads, and Faxén's theorem is applied to each of the beads. Faxén's theorem relates hydrodynamic forces and velocities of spherical objects immersed in arbitrary fluid flow fields. ${ }^{29}$ Analyzing the resulting relation between the bead velocities and the hydrodynamic forces on the beads for very long, thin rods (similar to Ref. 29), the following expression for the translational velocity is obtained:

$$
\mathbf{v}_{j}=-D_{t} \mathbf{D}\left(\hat{\mathbf{u}}_{j}\right) \cdot\left[\beta \nabla_{j} \Psi+\nabla_{j} \ln P\right]+\mathbf{U}\left(\mathbf{r}_{j}\right),
$$

with $\beta=1 / k_{B} T$, and

$$
D_{t}=\frac{k_{B} T \ln (L / D)}{4 \pi \eta_{0} L},
$$

which is proportional to the orientationally averaged translational diffusion coefficient for a free, noninteracting rod, with $L$ the length and $D$ the diameter of the rod, and $\eta_{0}$ the viscosity of the solvent. Furthermore,

$$
\mathbf{D}(\hat{\mathbf{u}}) \equiv \hat{\mathbf{I}}+\hat{\mathbf{u}} \mathbf{u},
$$

while $\Psi$ in Eq. (4) is the total potential energy and $\mathbf{U}$ is the suspension flow velocity. The rotational velocity is found similarly and equals

$$
\boldsymbol{\Omega}_{j}=-D_{r}\left[\beta \hat{\mathcal{R}}_{j} \Psi+\hat{\mathcal{R}}_{j} \ln P\right]+\hat{\mathbf{u}}_{j} \times\left[\hat{\mathbf{u}}_{j} \cdot \nabla_{j} \mathbf{U}\left(\mathbf{r}_{j}\right)\right],
$$

with

$$
D_{r}=\frac{3 k_{B} T \ln (L / D)}{\pi \eta_{0} L^{3}}
$$

the rotational diffusion coefficient for a free, noninteracting rod.

In both Eqs. (4) and (5) the total potential energy accounts for direct interactions between the rods. The total potential energy is now assumed to be pairwise additive, that is,

$$
\Psi\left(\mathbf{r}_{1}, \ldots, \mathbf{r}_{N}, \hat{\mathbf{u}}_{1}, \ldots, \hat{\mathbf{u}}_{N}\right)=\sum_{i<j} V\left(\mathbf{r}_{i}-\mathbf{r}_{j}, \hat{\mathbf{u}}_{i}, \hat{\mathbf{u}}_{j}\right),
$$

where $V$ is the interaction potential for two isolated rods: the "pair-interaction potential." In case of excluded volume in- teractions this is an exact relation. Integration of the Smulochowski equation (3) with respect to $\mathbf{r}_{1}, \mathbf{r}_{2}, \ldots, \mathbf{r}_{N}$ and $\hat{\mathbf{u}}_{2}, \ldots, \hat{\mathbf{u}}_{N}$, after the substitution of Eqs. (4) and (5), results in the equation of motion for the reduced pdf $P(\hat{\mathbf{u}}, t)$ for the orientation $\hat{\mathbf{u}}$ of a rod. This equation of motion depends on the two-particle pdf $P\left(\mathbf{r}, \mathbf{r}^{\prime}, \hat{\mathbf{u}}, \hat{\mathbf{u}}^{\prime}, t\right)$. In order to obtain a closed equation of motion, let us first introduce the paircorrelation function $g$. For a homogeneous system the paircorrelation function is defined as

$$
\begin{aligned}
P\left(\mathbf{r}, \mathbf{r}^{\prime}, \hat{\mathbf{u}}, \hat{\mathbf{u}}^{\prime}, t\right) \equiv & \int d \mathbf{r}_{3} \ldots \int d \mathbf{r}_{N} \oint d \hat{\mathbf{u}}_{3} \ldots \oint d \hat{\mathbf{u}}_{N} \\
& \times P\left(\mathbf{r}, \mathbf{r}^{\prime}, \mathbf{r}_{3}, \ldots, \mathbf{r}_{N}, \hat{\mathbf{u}}, \hat{\mathbf{u}}^{\prime}, \hat{\mathbf{u}}_{3}, \ldots, \hat{\mathbf{u}}_{N}, t\right) \\
\equiv & \frac{1}{V^{2}} P(\hat{\mathbf{u}}, t) P\left(\hat{\mathbf{u}}^{\prime}, t\right) g\left(\mathbf{r}, \mathbf{r}^{\prime}, \hat{\mathbf{u}}, \hat{\mathbf{u}}^{\prime}, t\right) .
\end{aligned}
$$

We shall approximate the pair-correlation function as

$$
g\left(\mathbf{r}, \mathbf{r}^{\prime}, \hat{\mathbf{u}}, \hat{\mathbf{u}}^{\prime}, t\right)=\exp \left(-\beta V\left(\mathbf{r}-\mathbf{r}^{\prime}, \hat{\mathbf{u}}, \hat{\mathbf{u}}^{\prime}\right)\right) .
$$

This expression for $g$, when used to close the Smoluchowski equation, reproduces the Doi-Edwards ${ }^{19}$ equation of motion, which is know to reproduce the Onsager free energy functional ${ }^{15}$ for long thin rods, in the absence of shear flow, which is exact in the limit where $D / L \rightarrow 0$. In principle, $g$ will be affected by shear flow. However, the effect of shear flow on the one-particle pdf $P(\hat{\mathbf{u}}, t)$ is probably much more important.

Substitution of Eqs. (4) and (5) into Eq. (3), and then performing the integration as described earlier, one finds

$$
\begin{aligned}
\frac{\partial}{\partial t} P(\hat{\mathbf{u}}, t)= & D_{r} \hat{\mathcal{R}} \cdot(\hat{\mathcal{R}} P(\hat{\mathbf{u}}, t)-\beta P(\hat{\mathbf{u}}, t) \overline{\mathbf{T}}(\hat{\mathbf{u}}, t)) \\
& -\hat{\mathcal{R}} \cdot P(\hat{\mathbf{u}}, t) \hat{\mathbf{u}} \times(\hat{\mathbf{u}} \cdot \nabla \mathbf{U}(\mathbf{r}, t)),
\end{aligned}
$$

where $\overline{\mathbf{T}}(\hat{\mathbf{u}}, t)$ the average torque on a rod, which is equal to

$$
\begin{aligned}
\overline{\mathbf{T}}(\hat{\mathbf{u}}, t)= & -\bar{\rho} \int d \mathbf{r}^{\prime} \oint d \hat{\mathbf{u}}^{\prime} P\left(\hat{\mathbf{u}}^{\prime}, t\right) g\left(\mathbf{r}-\mathbf{r}^{\prime}, \hat{\mathbf{u}}, \hat{\mathbf{u}}^{\prime}, t\right) \\
& \times \hat{\mathcal{R}} V\left(\mathbf{r}-\mathbf{r}^{\prime}, \hat{\mathbf{u}}, \hat{\mathbf{u}}^{\prime}\right)
\end{aligned}
$$

with $\bar{\rho}=N / V$ the number density. To within the approximation Eq. (7) for the pair-correlation function, we have, for excluded volume interactions,

$g\left(\mathbf{r}-\mathbf{r}^{\prime}, \hat{\mathbf{u}}, \hat{\mathbf{u}}^{\prime}\right) \hat{\mathcal{R}} V\left(\mathbf{r}-\mathbf{r}^{\prime}, \hat{\mathbf{u}}, \hat{\mathbf{u}}^{\prime}\right)=\beta^{-1} \chi\left(\mathbf{r}-\mathbf{r}^{\prime}, \hat{\mathbf{u}}, \hat{\mathbf{u}}^{\prime}\right)$,

where $\chi$ is the characteristic function for core-overlap, that is, $\chi=1$ when the cores of the rods overlap and is 0 otherwise. Since for very long, thin rods,

$$
\int d \mathbf{r}^{\prime} \chi\left(\mathbf{r}-\mathbf{r}^{\prime}, \hat{\mathbf{u}}, \hat{\mathbf{u}}^{\prime}\right)=2 D L^{2}\left|\hat{\mathbf{u}} \times \hat{\mathbf{u}}^{\prime}\right|
$$

the average torque is found to be equal to

$$
\overline{\mathbf{T}}(\hat{\mathbf{u}}, t)=-2 \beta^{-1} D L^{2} \bar{\rho} \hat{\mathcal{R}} \oint d \hat{\mathbf{u}}^{\prime} P(\hat{\mathbf{u}}, t)\left|\hat{\mathbf{u}} \times \hat{\mathbf{u}}^{\prime}\right| .
$$

We shall expand the right-hand side of this equation to fourth order in bilinear products of the orientations $\hat{\mathbf{u}}$ and $\hat{\mathbf{u}}^{\prime}$. This Ginzburg-Landau type of expansion results in a fourth order expansion of the free energy with respect to $\mathbf{S}$, in the 
case where no shear flow is applied. Such an expansion captures the essential physics. Hence, we shall approximate, $\mid \hat{\mathbf{u}}$ $\times \hat{\mathbf{u}}^{\prime} \mid \approx 1-\frac{1}{2}\left(\hat{\mathbf{u}} \cdot \hat{\mathbf{u}}^{\prime}\right)^{2}$, which is reminiscent to the well known Maier-Saupe approximation for the pair-interaction potential for two hard rods. ${ }^{30-32}$ Evaluation of the average torque now results in

$$
\overline{\mathbf{T}}(\hat{\mathbf{u}}, t)=2 \beta^{-1} D L^{2} \bar{\rho} \hat{\mathbf{u}} \times[\mathbf{S}(t) \cdot \hat{\mathbf{u}}] .
$$

Multiplying both sides of Eq. (8) by ûû, using Eq. (13), and then integrating with respect to $\hat{\mathbf{u}}$, yields an equation of motion for the order parameter tensor $\mathbf{S}$. This result is only valid for the homogeneous system, where the shear-rate, the density, and orientational order are independent of position,

$$
\begin{aligned}
\frac{\partial}{\partial t} \mathbf{S}= & -2 D_{r}\left[3 \mathbf{S}-\hat{\mathbf{I}}-2 D L^{2} \overline{\boldsymbol{\rho}}\left(\mathbf{S} \cdot \mathbf{S}-\mathbf{S}^{(4)}: \mathbf{S}\right)\right] \\
& +\dot{\gamma}\left[\hat{\boldsymbol{\Gamma}} \cdot \mathbf{S}+\mathbf{S} \cdot \hat{\boldsymbol{\Gamma}}^{T}-2 \mathbf{S}^{(4)}: \hat{\boldsymbol{\Gamma}}\right]
\end{aligned}
$$

where

$$
\mathbf{S}^{(4)}=\langle\hat{\mathbf{u} u ̂ u ̂ u}\rangle
$$

is a fourth order tensor. To be able to evaluate Eq. (14), $\mathbf{S}^{(4)}$ must be expressed in terms of $\mathbf{S}$. Following Hinch, ${ }^{33}$ such a closure relation can be constructed by noting that the degree of alignment of the rods is a monotonically increasing function of the shear-rate. An interpolation between the known forms of $\mathbf{S}^{(4)}$ for the isotropic and the perfectly aligned states is therefore expected to be reasonably accurate. Assuming a second order polynomial in $\mathbf{S}$ for $\mathbf{S}^{(4)}$, a calculation of the coefficients from the known forms for the isotropic and fully aligned states, and imposing trivial contraction properties, yields, for arbitrary tensors $\mathbf{M}$,

$$
\begin{aligned}
& \langle\hat{\mathbf{u}} \hat{u} \hat{u} \hat{u}\rangle: \mathbf{M}=\frac{1}{5}\left[\mathbf{S} \cdot \mathbf{M}_{S}+\mathbf{M}_{S} \cdot \mathbf{S}-\mathbf{S} \cdot \mathbf{S} \cdot \mathbf{M}_{S}-\mathbf{M}_{S} \cdot \mathbf{S} \cdot \mathbf{S}\right. \\
& \left.+2 \mathbf{S} \cdot \mathbf{M}_{S} \cdot \mathbf{S}+3 \mathbf{S S}: \mathbf{M}_{S}\right],
\end{aligned}
$$

with

$$
\mathbf{M}_{S}=\frac{1}{2}\left[\mathbf{M}+\mathbf{M}^{T}\right]
$$

the symmetric part of $\mathbf{M}$. Using this closure relation in Eq. (14), we finally find,

$$
\begin{aligned}
\frac{\partial}{\partial t} \mathbf{S}= & -\dot{\gamma}\left(\frac{2}{5}[\mathbf{S} \cdot \hat{\mathbf{E}}+\hat{\mathbf{E}} \cdot \mathbf{S}-\mathbf{S} \cdot \mathbf{S} \cdot \hat{\mathbf{E}}-\hat{\mathbf{E}} \cdot \mathbf{S} \cdot \mathbf{S}+2 \mathbf{S} \cdot \hat{\mathbf{E}} \cdot \mathbf{S}+3 \mathbf{S S}: \hat{\mathbf{E}}]\right. \\
& \left.-\hat{\boldsymbol{\Gamma}} \cdot \mathbf{S}-\mathbf{S} \cdot \hat{\boldsymbol{\Gamma}}^{T}\right)-D_{r}\left[6 \mathbf{S}-2 \hat{\mathbf{I}}-\frac{12}{5} D L^{2} \overline{\boldsymbol{\rho}}(\mathbf{S} \cdot \mathbf{S}-\mathbf{S S}: \mathbf{S})\right],
\end{aligned}
$$

with

$$
\hat{\mathbf{E}}=\frac{1}{2}\left[\hat{\boldsymbol{\Gamma}}+\hat{\boldsymbol{\Gamma}}^{T}\right] .
$$

This is the equation of motion that is at the basis of our interpretation of experimental data and the calculation of the phase diagram as far as the spinodals are concerned.

As can be seen from the above derivation, the DoiEdwards equation is obtained when assuming that the paircorrelation function has the form as given in Eq. (7), that is, when higher order correlations are neglected. For very long, thin rods this is probably a good approximation. Furthermore the translational and rotational diffusion coefficients relate to diffusion of a single, noninteracting rod, and are therefore independent of concentration. The concentration dependence of diffusion coefficients can in principle be calculated from the Smoluchowski equation, similar to calculations for spherical colloids (see for example Ref. 29). The extension of the above analysis of the Smoluchowski equation to inhomogeneous systems is currently under investigation [J. K. G. Dhont, W. J. Briels, to be published].

\section{The shear-rate dependent spinodals}

Let us first define the effective rotational diffusion coefficient $D_{r}^{\text {eff }}$ as follows. Let $\mathbf{S}_{0}$ denote a stationary solution of the equation of motion Eq. (16). Now write $\mathbf{S}(t)=\mathbf{S}_{0}$ $+\delta \mathbf{S}(t)$, and linearize the equation of motion Eq. (16) with respect to $\delta \mathbf{S}(t)$. The linearized equation of motion will be of the form

$$
\frac{\partial \delta \mathbf{S}(t)}{\partial t}=-c \mathbf{D}_{r}^{\mathrm{eff}} \cdot \delta \mathbf{S}(t),
$$

with $c$ a numerical constant that is chosen such that for infinite dilution and in the absence of shear flow, the effective diffusion tensor $\mathbf{D}_{r}^{\text {eff }}$ becomes equal to $D_{r} \hat{\mathbf{I}}$, where $D_{r}$ is the single particle diffusion coefficient. The effective rotational diffusion tensor depends on concentration and on $\mathbf{S}_{0}$. When at least one eigenvalue of $\mathbf{D}_{r}^{\text {eff }}$ is negative, the stationary solution $\mathbf{S}_{0}$ is unstable. By definition (see Sec. II B) the density and the state $\mathbf{S}_{0}$ are on the spinodal when the smallest eigenvalue of $\mathbf{D}_{r}^{\text {eff }}$ is zero. The rotational dynamics are thus extremely slow close to the spinodal (this is analogous to critical slowing of translational diffusion in case of a gasliquid transition). The spinodal concentration where the isotropic state without shear flow becomes absolutely unstable can be found by linearization around $\mathbf{S}_{0}=\frac{1}{3} \hat{\mathbf{I}}$. Hence we write

$$
\mathbf{S}(t)=\frac{1}{3} \hat{\mathbf{I}}+\delta \mathbf{S}(t),
$$

and linearize Eq. (16) with respect to $\delta \mathbf{S}(t)$. The solution of the resulting linear equation of motion is

$$
\delta \mathbf{S}(t)=\delta \mathbf{S}(t=0) \exp \left\{-6 D_{r}^{\mathrm{eff}} t\right\},
$$

where the effective rotational diffusion coefficient is given by

$$
D_{r}^{\mathrm{eff}}=D_{r}\left(1-\frac{2}{15} D L^{2} \bar{\rho}\right) .
$$

For concentrations where $\frac{2}{15} D L^{2} \rho>1$, the effective rotational diffusion coefficient is negative, so that Eq. (19) predicts an exponential increase of $\delta \mathbf{S}(t)$ with time. The spinodal concentration for the isotropic-to-nematic transition, in terms of the volume fraction $\varphi$ of rods, is thus found to be equal to $(L / D) \varphi=5.89$. This number should be compared to its exact value, 4, for very long, thin rods as found by Onsager. ${ }^{15}$ The discrepancy between our result and the exact value is primarily due to the Ginzburg-Landau type of expansion, and to a lesser extent, to the closure relation.

In the case where shear flow is applied it is convenient to introduce the dimensionless rotational Peclet number, 


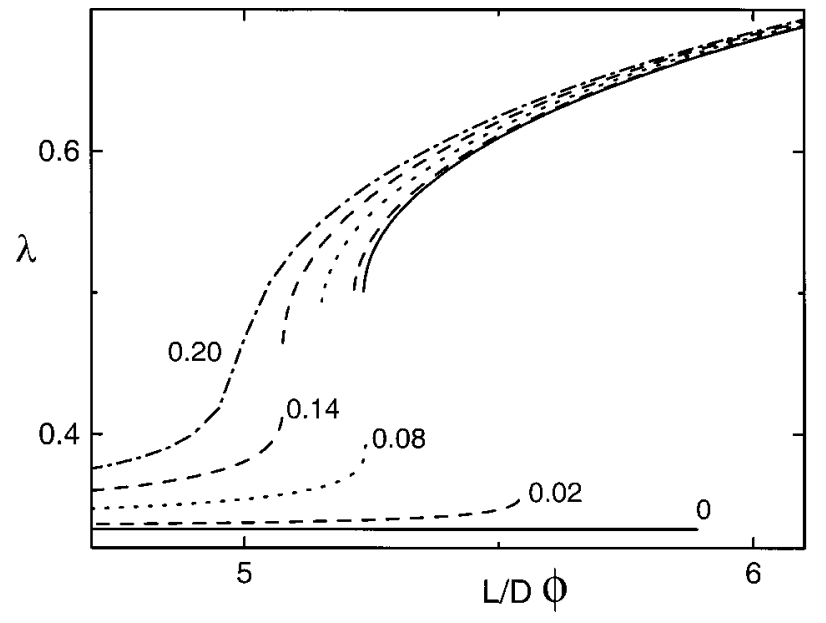

FIG. 2. The largest eigenvalue of $\mathbf{S}$ vs concentration. The lines represent stable solutions of the equation of motion [Eq. (16)], for various bare Peclet numbers as indicated in the figure.

$$
\mathrm{Pe}_{r}^{0}=\dot{\gamma} / D_{r}
$$

and the dimensionless time

$$
\tau=D_{r} t .
$$

The rotational Peclet number measures the effect of the shear flow on orientational microstructure in the case where the effective diffusion coefficient is not very different from the bare diffusion coefficient $D_{r}$. The stationary state under shear flow around which one should linearize the equation of motion Eq. (16) is not known analytically, but must be found numerically. Bifurcation diagrams, where the largest eigenvalue $\lambda$ of the order parameter tensor $\mathbf{S}$ for stable states is plotted versus $(L / D) \varphi$, are collected in Fig. 2 for various shear-rates. Diagrams of this sort are extensively discussed in Refs. 34, 35. The curves in the diagram are attractors for the equation of motion Eq. (16) for a given Peclet number $\mathrm{Pe}_{r}^{0}$. The spinodal points are the points where the curves terminate: here the attractor becomes a repellor. As can be seen, for small enough shear-rates there is a concentration range where there are two stable solutions of Eq. (16): a so-called paranematic state (the isotropic state which is slightly shear-aligned) and a nematic state. These concentrations are within the two-phase region. Above a certain critical shear-rate, corresponding to a Peclet number equal to $\mathrm{Pe}_{r, c}^{0} \approx 0.15$, the paranematic and the nematic branches merge into one single curve. For these large shear-rates, thermodynamic forces are too small in comparison to shear forces to be able to induce a discontinuous phase transition. The nonequilibrium phase diagram, as far as the spinodals are concerned, is given in Fig. 3.

\section{E. The relation between birefringence and the orientational order parameter tensor}

Before describing the experimental method to obtain the shear-rate dependent spinodals, we shall have to establish the relation between birefringence and the orientational order parameter tensor. Let $\epsilon_{\|}$and $\epsilon_{\perp}$ denote the dielectric constants for polarization directions of the light parallel and perpen-

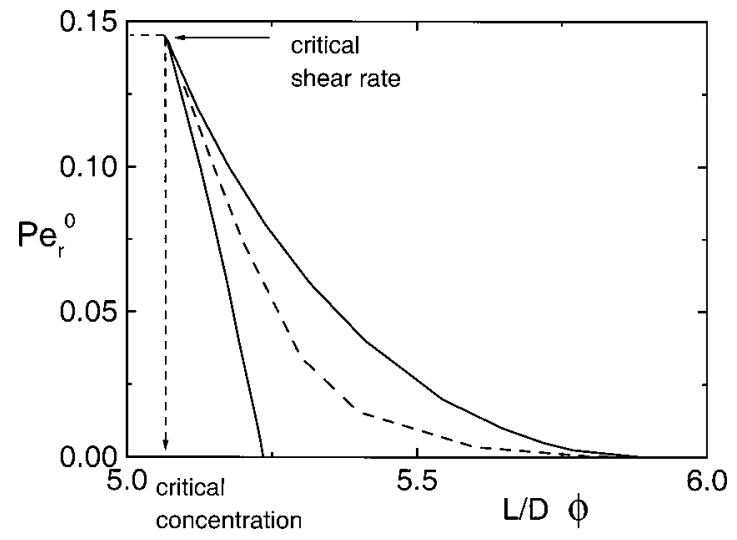

FIG. 3. Nonequilibrium spinodals. The lower solid line is the spinodal where the nematic phase becomes unstable on lowering the concentration (the nematic-to-paranematic spinodal), while the upper solid line is the spinodal where the paranematic phase becomes unstable on increasing the concentration (the paranematic-to-nematic spinodal). The dotted line is the predicted experimental curve as obtained from birefringence hysteresis experiments, as calculated from the equation of motion Eq. (16) (see Sec. II F).

dicular to the rod's long axis, respectively. These values are assumed to be constant within the core of the rod. The difference between $\epsilon_{\|}$and $\epsilon_{\perp}$ comprises both intrinsic anisotropy of the rod material as well as "form contributions" due to intra-particle multiple scattering.

By decomposing an incident electric field into a component parallel to the unit vector $\hat{\mathbf{u}}$ that specifies the orientation of the long axis of a rod and a component perpendicular to it, the dielectric constant $\boldsymbol{\epsilon}_{\mathrm{rod}}$ of the rod is found to be equal to

$$
\boldsymbol{\epsilon}_{\mathrm{rod}}=\epsilon_{\|} \hat{\mathbf{u}} \hat{\mathbf{u}}+\boldsymbol{\epsilon}_{\perp}[\hat{\mathbf{I}}-\hat{\mathbf{u}} \hat{\mathbf{u}}]=\overline{\boldsymbol{\epsilon}} \hat{\mathbf{I}}+\Delta \epsilon\left[\hat{\mathbf{u}} \hat{\mathbf{u}}-\frac{1}{3} \hat{\mathbf{I}}\right],
$$

with

$$
\bar{\epsilon}=\frac{1}{3}\left(\epsilon_{\|}+2 \epsilon_{\perp}\right), \quad \Delta \epsilon=\epsilon_{\|}-\epsilon_{\perp} .
$$

The dielectric constant is now a tensorial quantity. The dielectric constant of the suspension is a linear combination of the dielectric constants of the solvent and the rod material. This is a very good approximation, certainly at the very low volume fractions of interest for the long and thin rods under consideration. The apparent dielectric constant of the suspension $\boldsymbol{\epsilon}$, relative to that of the solvent, is thus equal to (with $\epsilon_{f}$ the dielectric constant of the solvent and $\varphi$ the volume fraction of rods),

$$
\begin{aligned}
\boldsymbol{\epsilon} & =\varphi \boldsymbol{\epsilon}_{\mathrm{rod}}+(1-\varphi) \boldsymbol{\epsilon}_{f} \hat{\mathbf{I}} \\
& =\left[\varphi \overline{\boldsymbol{\epsilon}}+(1-\varphi) \boldsymbol{\epsilon}_{f}\right] \hat{\mathbf{I}}+\varphi \Delta \epsilon\left[\hat{\mathbf{u}} \hat{\mathbf{u}}-\frac{1}{3} \hat{\mathbf{I}}\right] \\
& \equiv \epsilon_{\mathrm{iso}} \hat{\mathbf{I}}+\varphi \Delta \epsilon \hat{\mathbf{u}} \hat{\mathbf{u}},
\end{aligned}
$$

with $\epsilon_{\text {iso }}$ the isotropic part of the dielectric tensor, which is equal to

$$
\epsilon_{\mathrm{iso}}=\varphi \bar{\epsilon}+(1-\varphi) \epsilon_{\perp}-\frac{1}{3} \varphi \Delta \epsilon=\varphi \epsilon_{\perp}+(1-\varphi) \epsilon_{f} .
$$

We assumed, in Eq. (24), that all rods are identical in optical properties. The apparent dielectric constant $\epsilon(\hat{\mathbf{n}})$ for a given polarization direction $\hat{\mathbf{n}}$ of the light is equal to $\hat{\mathbf{n}} \cdot \boldsymbol{\epsilon} \cdot \hat{\mathbf{n}}$. The corresponding macroscopic refractive index is equal to 


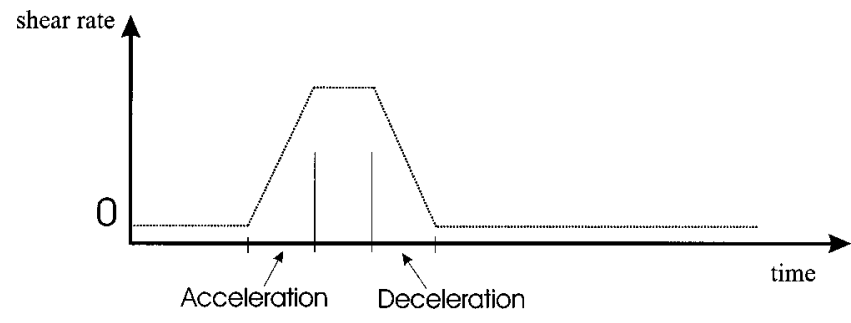

FIG. 4. A shear-rate sweep. The shear-rate is increased linearly in time during a time interval of 0.5 to $1 \mathrm{~s}$, after which the shear-rate is kept constant for $10 \mathrm{~s}$ (varying from 1 to $7 \mathrm{~s}^{-1}$ ) and then decreased linearly in time to 0 within the same time span.

$n_{m}(\hat{\mathbf{n}}) \sim\langle\sqrt{\boldsymbol{\epsilon}(\hat{\mathbf{n}})}\rangle$, where the brackets $\langle\ldots\rangle$ denote ensemble averaging with respect to orientation. To leading order in $\Delta \epsilon / \epsilon_{\text {iso }}$ we thus find

$$
n_{m}(\hat{\mathbf{n}})=n_{\text {iso }}\left[1+\frac{1}{2} \varphi \frac{\Delta \epsilon}{\epsilon_{\text {iso }}} \hat{\mathbf{n}} \cdot \mathbf{S} \cdot \hat{\mathbf{n}}\right],
$$

where $n_{\text {iso }} \sim \sqrt{\epsilon_{\text {iso }}}$ is the refractive index corresponding to the dielectric constant $\epsilon_{\text {iso }}$. The assumption here is that the magnetic susceptibility of the fluid and the rods are both equal to that of vacuum.

The birefringence $\Delta n^{\prime}$ is defined as the maximum difference in the refractive index $n_{m}(\hat{\mathbf{n}})$ on varying $\hat{\mathbf{n}}$ within the plane perpendicular to the propagation direction of the light beam. In our experiments, the light beam propagates along the gradient direction (the $y$-direction, see Fig. 1). Since for the geometry used here we have $\hat{\mathbf{n}}=\left(\mu, 0, \sqrt{1-\mu^{2}}\right)$, with $0 \leqslant \mu \leqslant 1$, it is found that $\hat{\mathbf{n}} \cdot \mathbf{S} \cdot \hat{\mathbf{n}}=\mu^{2}\left(S_{11}-S_{33}\right)+S_{33}$ (note that $S_{13}=0$, due to symmetry with respect to the $x y$-plane). Hence the maximum and minimum values of the refractive index are taken by $\mu=0$ and $\mu=1$, that is, for $\hat{\mathbf{n}}$ along the $x$ and $z$-direction, respectively. We thus find that

$$
\Delta n^{\prime} \sim c\left|S_{33}-S_{11}\right|,
$$

with $S_{i j}$ the $i j$ th component $\mathbf{S}$ and $c$ the concentration. This is a special case of the formula given by Fuller (Ref. 36, p. 113), with $S_{13}=0$.

\section{F. The experimental procedure}

The intuitive idea behind the procedure used to obtain the shear-rate dependent spinodals is the following. Consider a homogeneous system of rodlike particles with a concentration below the nematic-to-isotropic spinodal concentration $(L / D) \varphi=5.24$, and above the critical concentration (see Fig. 3 ). Suppose the system is subjected to a "shear-rate sweep" as depicted in Fig. 4: the shear-rate is increased with time during 0.5 to $1 \mathrm{~s}$ up to a value between $1-7 \mathrm{~s}^{-1}$, which is then maintained for some time, after which the shear-rate is decreased to zero. On increasing the shear-rate, the concentration where the paranematic phase becomes unstable decreases, as can be seen from the bifurcation diagram Fig. 2. At a certain shear-rate $\left(\dot{\gamma}_{1}\right.$, say), the paranematic-to-nematic spinodal concentration will be lower than the actual concentration. The system will then, initially very slowly, evolve toward the stable nematic state. On decreasing the shear-rate after some time, the nematic state will become unstable again at a certain shear-rate $\left(\dot{\gamma}_{2}\right.$, say). The system will then return to the paranematic state. Since $\dot{\gamma}_{1}>\dot{\gamma}_{2}$, as can be seen from the bifurcation diagrams in Fig. 2, there will be a hysteresis in the measured birefringence during such a shear-rate sweep. The shear-rate where the maximum hysteresis in birefringence occurs will be in between the two shear-rates $\dot{\gamma}_{1}$ and $\dot{\gamma}_{2}$ where the paranematic and nematic phase become unstable, respectively. On variation of the concentration, this procedure provides a line in the nonequilibrium phase diagram that is in between the two spinodals. Since rotational dynamics close to the spinodals are very slow, the form of the hysteresis curve will probably depend on the shear sweep rate, that is, the rate of change of the shear-rate. The effect of the very slow dynamics near the spinodal points can be eliminated by extrapolating hysteresis curves to a sweep rate equal to zero. For concentrations above the nematic-toisotropic spinodal concentration $(L / D) \varphi=5.24$, the system will tend toward the stable nematic state above a certain shear-rate, and will not return to the isotropic branch again on subsequent decrease of the shear-rate. There may be an exception when the system is not allowed to actually attain the stable nematic state before decreasing the shear-rate again, that is, when the state of the system remains in the basin of attraction of the paranematic branch during the entire experiment. These intuitive ideas will be quantified below on the basis of the equation of motion Eq. (16).

There are two assumptions made in the above reasoning. First of all it is assumed that phase separation under shear does not occur during a shear-rate sweep. For the fd system this was tested and no phase separation under shear flow was observed over a period of 2 days, while the birefringence experiments take about $5 \mathrm{~min}$. Second, it is assumed that the orientational order parameter is independent of position, that is, the possible existence of domains with different directors is ignored. In the absence of shear flow such domains will certainly exist, while in the presence of strong shear flow this is probably a reasonable assumption.

The shear-rate sweep that we employed in our experiments is as follows. The shear-rate is increased linearly in time during 0.5 to $1 \mathrm{~s}$ up to a certain final shear-rate. This time interval and the final shear-rate determine the shear sweep rate. The final shear-rate is maintained for $10 \mathrm{~s}$, after which the shear-rate is decreased again to 0 within the same time span (see Fig. 4).

To relate the shear-rate with the bare Peclet number [see Eq. (21)] and time with the dimensionless time [see Eq. (22)], we used the bare rotational diffusion coefficient of $20.9 \mathrm{~s}^{-1}$ as reported in Ref. 37. Hysteresis curves can be obtained from Eq. (16), where the shear-rate is now a prescribed function of time. Figure 5 shows plots of the hysteresis in birefringence, as obtained from Eq. (16), as a function of the actual bare Peclet number $P e_{r}^{0}$ during a shear-rate sweep.

Four different concentration regimes may be distinguished: concentrations below the critical concentration $((L / D) \varphi<5.07)$, concentrations in between the critical concentration and the nematic-to-isotropic spinodal (5.07 $<(L / D) \varphi<5.24)$, in between the nematic-to-isotropic spinodal and the isotropic-to-nematic spinodal $(5.24<(L / D) \varphi$ $<5.89)$, and larger concentrations $((L / D) \varphi>5.89)$. We 

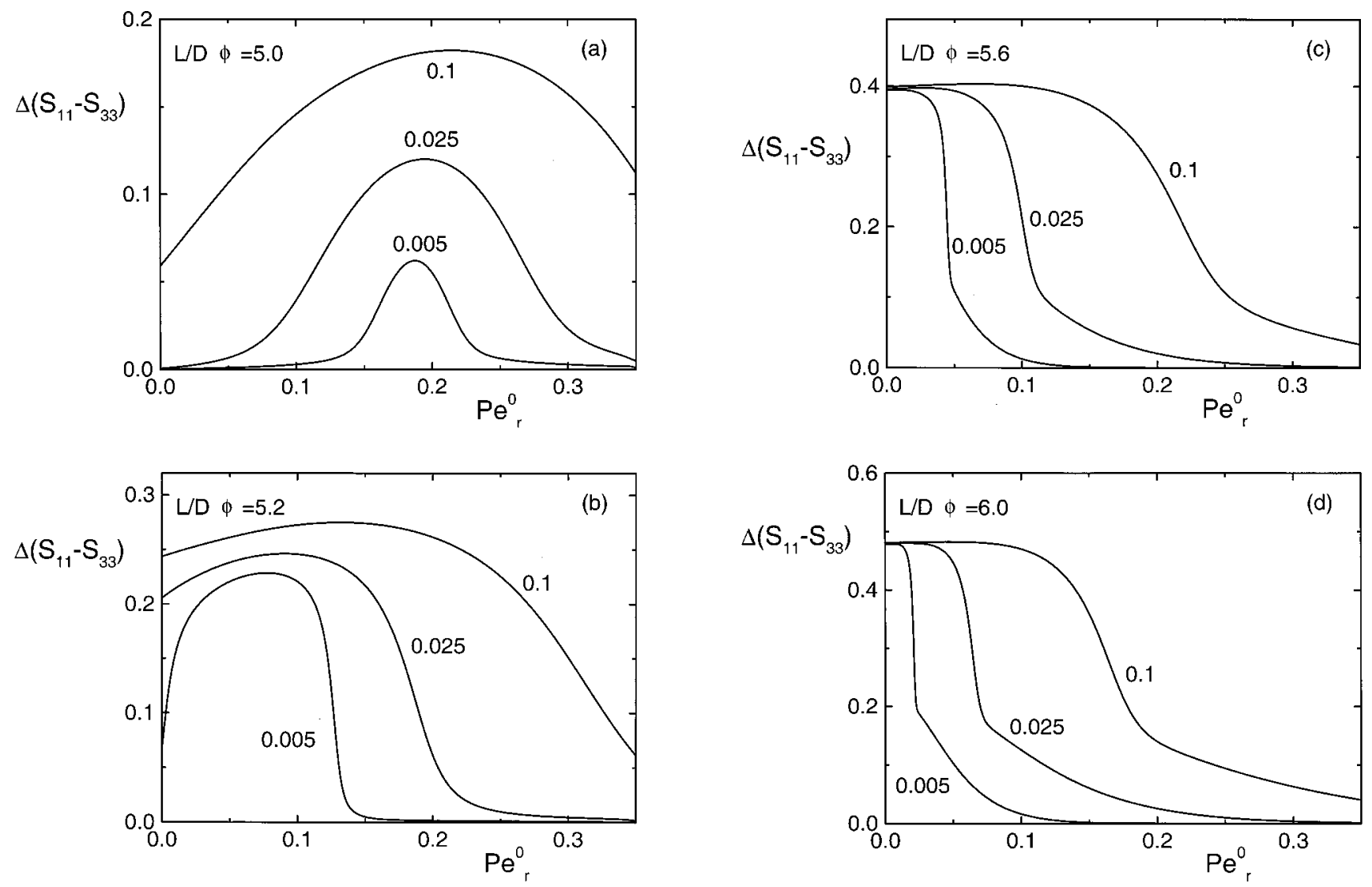

FIG. 5. Birefringence hysteresis curves [as calculated from Eq. (16)] for concentrations in the four different concentration regimes as defined in the main text. Plotted is the birefringence hysteresis $\Delta\left(S_{11}-S_{33}\right)$ [see Eq. (26)] vs the actual shear-rate during a shear-rate sweep, for different shear sweep rates. The shear sweep rates are indicated in the figures, and are defined here as $1 / \tau_{\text {end }}$, with $\tau_{\text {end }}$ the dimensionless time interval during which the maximum shear-rate is achieved in the acceleration period of the sweep (see Fig. 4).

shall refer to these concentration regimes as regimes I, II, III, and IV, respectively. Figures 5(a)-5(d) relate to these concentration regimes. As explained above, a clear maximum in the hysteresis curve is expected in regime II, and is indeed found in Fig. 5(b), since during a sweep, first the paranematic state becomes unstable on increasing the shear-rate and then the nematic state becomes unstable on decreasing the shear-rate. The maximum becomes much less pronounced in regime III [see Fig. 5(c)], since then the system attains the nematic state on increasing the shear-rate and remains in that state when the shear-rate is decreased, in accordance with our intuitive reasoning above.

Below the critical concentration, in regime I, the magnitude of the hysteresis goes to zero as the sweep rate is diminished, as can be seen in Fig. 5(a). For a finite sweep-rate there is a finite hysteresis because of the slow rotational dynamics close to the spinodals. At very small sweep-rates, the upward and downward measured birefringence curve will overlap, so that the birefringence hysteresis will disappear. Note, however, that the extrapolated shear-rate where the maximum hysteresis occurs still extrapolates to a finite value, which is indicative for the location of the critical shear-rate. For lower concentrations, further away from the spinodals, where rotational dynamics is relatively fast for all shear-rates, the magnitude of the hysteresis rapidly decreases to a value that is probably too small to be measured. For concentrations above the isotropic-to-nematic concentration, regime IV, the amplitude of the hysteresis remains large. However, its maximum value extrapolates to a zero shearrate, as can be seen in Fig. 5(d). This is due to the fact that one starts with a paranematic state, while after the sweep, the system remains in the nematic state, similar to what happens in regime III.

The Peclet number at which the hysteresis maximum is observed is plotted as a function of the sweep-rate in Fig. 6 for various concentrations. The values of this Peclet number, extrapolated to zero shear-rate, are plotted in Fig. 3 (the dashed line). As can be seen, this line lies in between the two spinodals, and is therefore an indication of how the location of the spinodal changes with shear-rate. Note that the critical shear-rate coincides with the shear-rate obtained from the above described experimental method.

On increasing the shear-rate during a sweep, one might expect a sudden increase in the birefringence once the paranematic-to-nematic spinodal point is crossed, and a subsequent sudden decrease on lowering the shear-rate when crossing the nematic-to-paranematic spinodal again. It turns out that the dynamics close to the spinodal points are so slow that an unambiguous identification of the shear-rate where the expected sudden increase or decrease occurs cannot be made accurately enough to really distinguish these from the shear-rate where the maximum in the hysteresis curve occurs. 


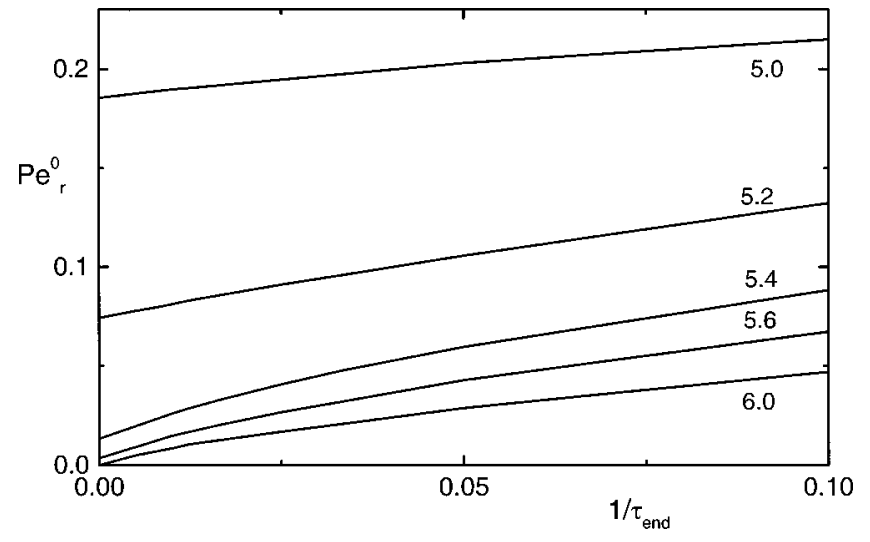

FIG. 6. The bare Peclet number at which the maximum hysteresis is found as a function of the sweep rate, with $\tau_{\text {end }}$ the dimensionless time during which the final shear-rate is reached. The numbers in the figure refer to the various concentrations in terms of $(L / D) \varphi$.

What is neglected in the above analysis is the existence of distinct nematic domains, which might be important for lower shear-rates. The order parameter tensor $\mathbf{S}$ is assumed to be the same everywhere in the system. It is unclear what the influence of the formation of inhomogeneties is on the measured birefringence.

\section{EXPERIMENTAL METHODS AND RESULTS}

\section{A. The colloidal system}

The system used in this study consists of the fd virus. These biopolymers have a contour length of $L=880 \mathrm{~nm}$ and a diameter $D=6.6 \mathrm{~nm}$, as obtained with electron microscopy. The persistence length of these molecules is $P$ $=2000 \mathrm{~nm}$, which is about twice the contour length. ${ }^{37} \mathrm{Fd}$ virus is therefore somewhat flexible. The solvent is a $10 \mathrm{mM}$ Tris(hydroxymethyl)-aminomethane buffer $(p \mathrm{H}=8.15)$ with $25 \mathrm{mM} \mathrm{NaCl}$ added. The $p \mathrm{H}$ was adjusted by adding a dilute solution of hydrochloric acid $(\mathrm{HCl})$ to the fd solution.

$\mathrm{Fd}$ virus molecules are charged, resulting in an effective diameter that is larger than the above mentioned thickness of $6.6 \mathrm{~nm}$. From Onsager's expression for the effective diameter of a charged rod $^{15}$ we obtain a diameter of $14.8 \mathrm{~nm}$. The effective aspect ratio is thus equal to $L / D=60$. Experimentally, the two binodal concentrations without shear flow are found to be equal to $13.0 \mathrm{mg} / \mathrm{ml}$ and $14.5 \mathrm{mg} / \mathrm{ml}$, respectively. These corresponds to values of $(L / D) \varphi$ of 4.5 and 4.8 , respectively (using a molar mass of $1.64 \times 10^{7} \mathrm{~g}$ ). ${ }^{37}$ These numbers are higher than the respective predicted values of 3.3 and 4.2 by Onsager. ${ }^{15}$ This discrepancy is probably due to the slight flexibility of fd virus and the tendency of the rods to acquire mutually perpendicular orientations due to their charges. ${ }^{37}$

\section{B. Experimental setup}

The home-built birefringence setup is based on the design by Fuller. ${ }^{36}$ The rotating half-wave plate is mounted on a dentist drill, which can achieve a rotational speed of about $4 \mathrm{kHz}$. The shear cell is a home-built optical Couette cell with a gapwidth of $2.47 \mathrm{~mm}$, which is placed in a thermo- stating, optical matching bath. The largest shear-rate that is applied is $7 \mathrm{~s}^{-1}$. The acceleration and deceleration times during a shear-rate sweep varied from $0.5 \mathrm{~s}$ to $1.0 \mathrm{~s}$ (see Fig. 4). The direction of the laser beam relative to the shear geometry is depicted in Fig. 1.

\section{Shear alignment of fd virus}

Before discussing the shear induced shift of spinodals as obtained by the above described hysteresis measurements, flow alignment experiments will be presented in the following subsection. As will be seen, there is qualitative agreement between experiment and theory, which validates the use of Eq. (16) for the interpretation of the experimental results to obtain the shear-induced shift of spinodals. Due to the Ginzburg-Landau type of expansion that was employed to derive the equation of motion Eq. (16), and the neglect of defects, there is only qualitative agreement.

Flow alignment experiments are performed on suspensions of fd virus for shear-rates varying from 1 and $7 \mathrm{~s}^{-1}$, and concentrations varying from $3.5 \mathrm{mg} / \mathrm{ml}$ (the overlap concentration is $0.076 \mathrm{mg} / \mathrm{ml}$ ), up to $14.05 \mathrm{mg} / \mathrm{ml}$, which is close to the binodal nematic concentration. Figure 7 shows typical birefringence curves, where the shear-rate is linearly increased from 0 to its final value within the time interval 5 to $6 \mathrm{~s}$, and linearly decreased again to 0 in the time interval 16 to $17 \mathrm{~s}$.

As expected, birefringence increases with increasing shear-rate and increasing concentration. In Fig. 7, numerical results obtained from Eq. (16) are plotted in the insets. The proportionality constant in Eq. (26), is chosen so as to match the birefringence level for the highest concentration. The proportionality constant that appears on the right-hand side of Eq. (26), is found to be equal to $0.33 \times 10^{-4}$, when the concentration $c$ is expressed in $\mathrm{mg} / \mathrm{ml}$. Mapping of the concentration in $\mathrm{mg} / \mathrm{ml}$ onto $(L / D) \varphi$ can be done by relating the spinodal concentrations found from Eq. (16) and the experimentally found binodal concentrations with Onsager's exact results. Since the coexistence region for fd virus suspensions is much narrower than predicted by Onsager, there is no unambiguous choice for the proportionality constant between values of $(L / D) \varphi$ and concentrations in units of $\mathrm{mg} / \mathrm{ml}$. We chose a value of $0.38 \mathrm{ml} / \mathrm{mg}$ for the proportionality constant so as to have an optimum match between the experimental shear alignment experiments and the theoretical prediction based on Eq. (16). For the calculation of the bare rotational Peclet number and the dimensionless time we used a bare rotational diffusion coefficient of $11 \mathrm{~s}^{-1}$. This value of the rotational diffusion coefficient is found by comparing the critical shear-rates as obtained from Eq. (16) with the experimentally obtained critical shear-rate (see Fig. 12). The difference with the value of $20.9 \mathrm{~s}^{-1}$ that was found in Ref. 37 is probably due to the approximate nature of our theory and differences in contributions from electrolyte friction. ${ }^{38}$ As can be seen, the experimental birefringence curves are in qualitative agreement with theory, except that for the highest concentrations, theory predicts no decay of the birefringence to 0 . For these higher concentrations the system remains in the stable nematic state after cessation of the shear flow. The experimentally observed relaxation of birefringence is prob- 

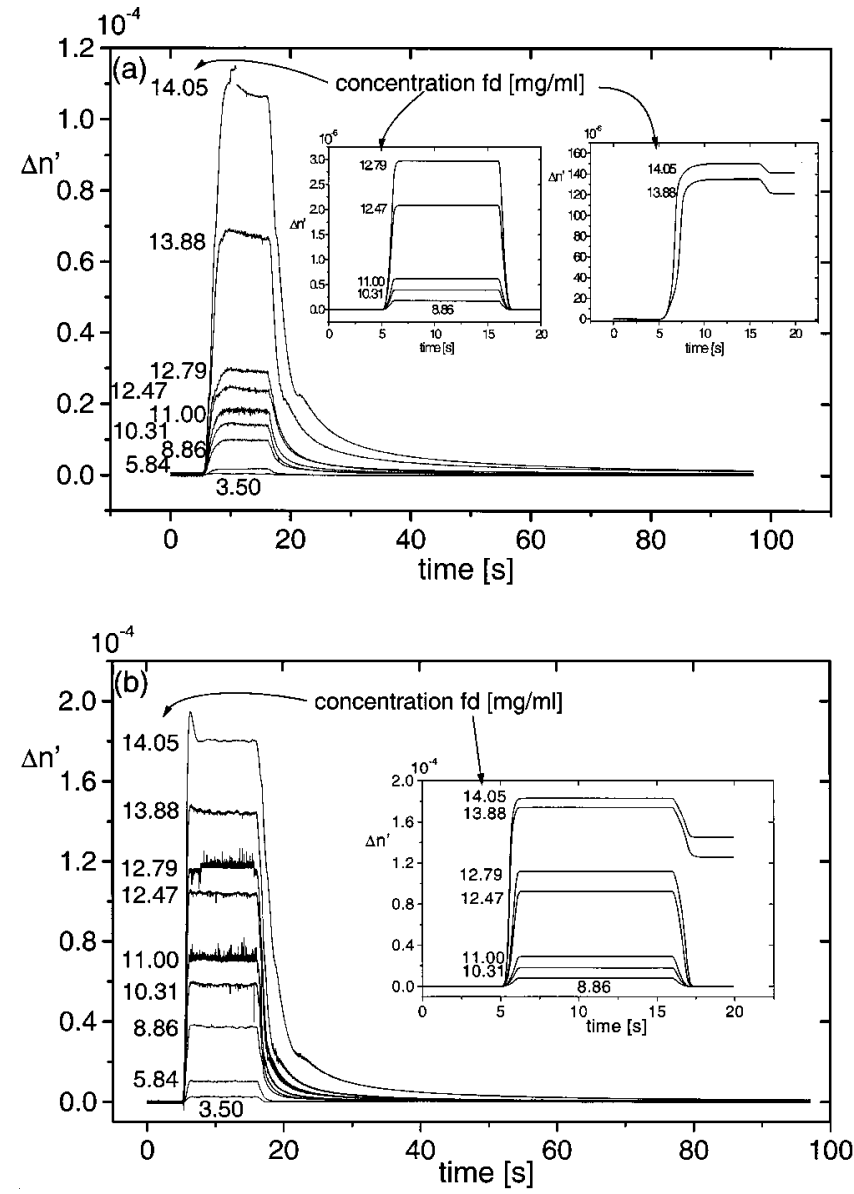

FIG. 7. Birefringence as a function of time, where the shear-rate is linearly increased between 5 and $6 \mathrm{~s}$, and decreased to 0 again between 16 and $17 \mathrm{~s}$ after the start of the measurement. In the time interval 6 to $16 \mathrm{~s}$, the shearrate is kept constant. (a) is for a final shear-rate of $1 \mathrm{~s}^{-1}$ and (b) for $7 \mathrm{~s}^{-1}$. The numbers in the figures refer to concentrations in $\mathrm{mg} / \mathrm{ml}$. The insets in both plots are numerical results as obtained from the equation of motion Eq. (16). The proportionality constant in Eq. (26) is determined from the stationary experimental birefringence level for the highest concentration.

ably due to the formation of domains with different directors. The formation of such inhomogeneities has been neglected in our theory. No more than qualitative agreement can be expected, due to the approximate nature of the GinzburgLandau type of expansion that was used.

Note the overshoot in birefringence in Figs. 7(a) and 7(b) that occurs only for concentrations which are close to the nematic binodal concentration. This effect is more pronounced for larger shear-rates. It is unclear what the precise microscopic origin of the overshoot is. As can be seen from Fig. 7, this overshoot is not predicted by theory.

The concentration dependence of the stationary value of the birefringence for four different shear-rates is shown in Fig. 8. A strong nonlinear increase of the birefringence in the neighborhood of the binodal concentration $(14.5 \mathrm{mg} / \mathrm{ml})$ is found. For these larger concentrations, thermodynamic forces assist the shear forces to align the rods. Note the almost linear increase of birefringence with concentration for the lowest shear-rate of $1 \mathrm{~s}^{-1}$, up to about $13.00 \mathrm{mg} / \mathrm{ml}$, beyond which thermodynamic forces render a rapid increase. The inset in Fig. 8 is the theoretical prediction from Eq. (16) for the same shear-rates as in the experiments. Theory un-

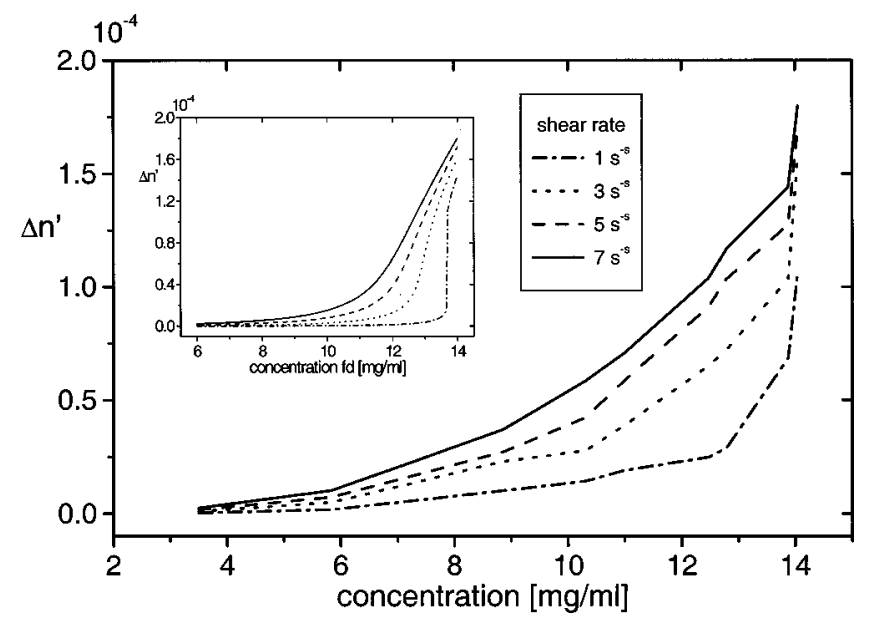

FIG. 8. Birefringence as a function of concentration for four shear-rates, which are indicated in the figure. The inset shows the theoretical prediction according to Eq. (16).

derestimates the birefringence at somewhat lower concentrations. The critical shear-rate for the fd virus suspension is 1.6 $\mathrm{s}^{-1}$ (see the subsequent subsection). Only the smallest shearrate in Fig. 8 is below this critical shear-rate. For larger shear-rates the birefringence is therefore expected to change continuously as a function of concentration. For the smallest shear-rate, the theoretically found concentration dependence exhibits a discontinuity where the paranematic state becomes unstable, and the systems jumps to the nematic state. The resolution in concentration for the data points in Fig. 8 is too crude to really see this discontinuity.

The shear-rate dependence of stationary birefringence is shown in Fig. 9 for various concentrations. The inset shows theoretical results obtained from Eq. (16). For the two highest concentrations, theory predicts a jump discontinuity at low shear-rates, where the system jumps from the paranematic branch to the nematic branch.

In all the above described experiments, we found qualitative agreement between experiment and theory. This validates the use of the theory described in Sec. II C for the interpretation of the experimental procedure described in

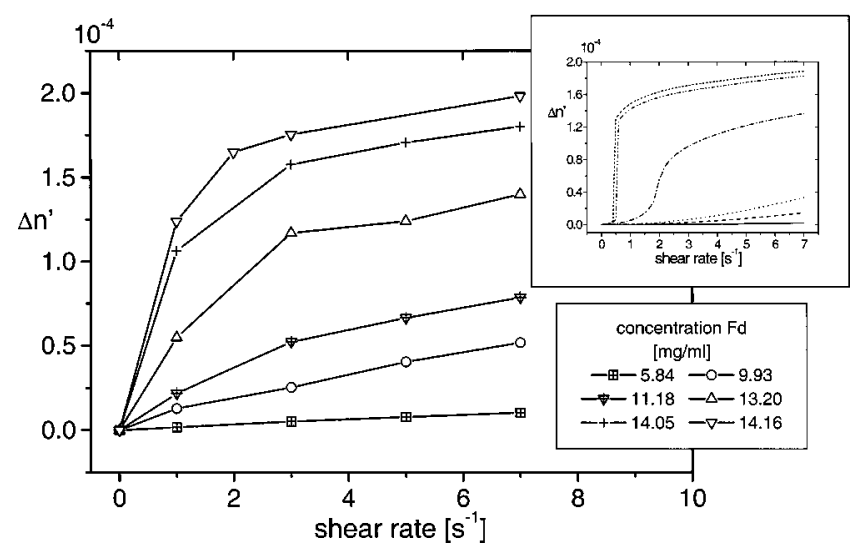

FIG. 9. Birefringence as a function of shear-rate for various concentrations, as indicated in the figure. The inset shows theoretical results as obtained from Eq. (16). 

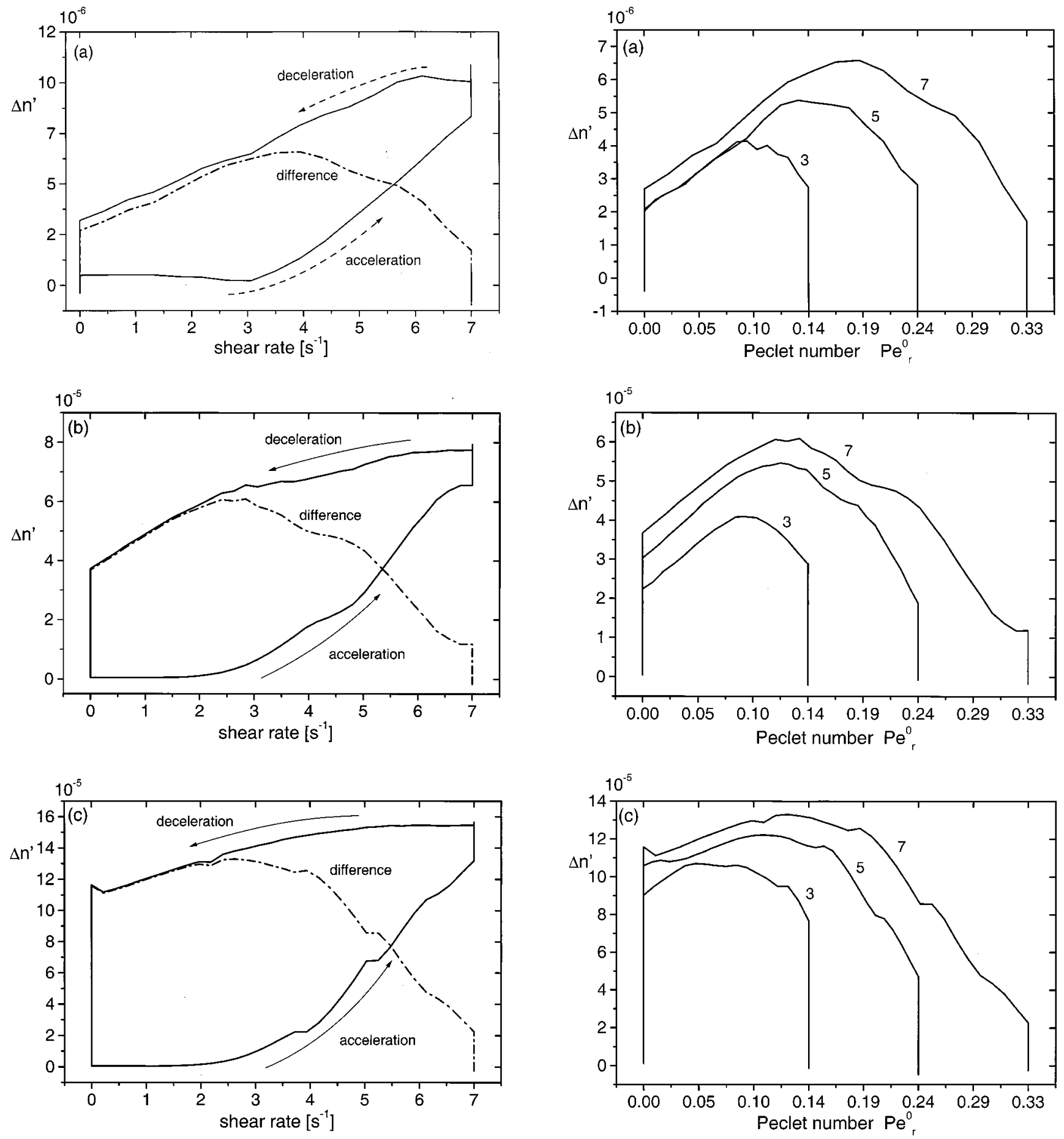

FIG. 10. Left column of figures: birefringence as a function of the actual shear-rate during a sweep (solid lines) and the hysteresis (dotted line), for four concentrations: (a) 5.84 , (b) 11.18 , and (c) $13.88 \mathrm{mg} / \mathrm{ml}$. The rate of change of the shear-rate is $7 \mathrm{~s}^{-2}$ (corresponding to the theoretically defined sweep rate $1 / \tau_{\text {end }}=0.335$, for a bare rotational diffusion coefficient of $20.9 \mathrm{~s}^{-1}$ ), while the highest shear-rate is $7 \mathrm{~s}^{-1}$ (corresponding to a bare rotational Peclet number of 0.33 ). Right column of figures: hysteresis curves for three different sweep rates. The numbers in the figure refer to sweep rates $d \dot{\gamma} / d t$ in units of $\mathrm{s}^{-2}$.

Sec. II F in order to obtain the shear-rate induced shift of spinodals.

It might be that there is a connection between slowing down of effective rotational diffusion and shear thinning. This is a subject of further research.

\section{Hysteresis experiments}

The experimentally applied time dependence of the shear-rate during a sweep is sketched in Fig. 4. The corre- sponding time dependence of the measured birefringence during a sweep (solid lines) and the difference between the two (dotted line) is plotted in Fig. 10 for three different concentrations; $5.84,11.18$, and $13.88 \mathrm{mg} / \mathrm{ml}$.

For the lowest concentration [Fig. 10(a)], the amplitude of the hysteresis curve is found to be much smaller than for the higher concentrations. This is in accordance with the theoretical prediction in Fig. 5, pertaining to the concentration regime I as defined in Sec. II F. The experimentally observed 

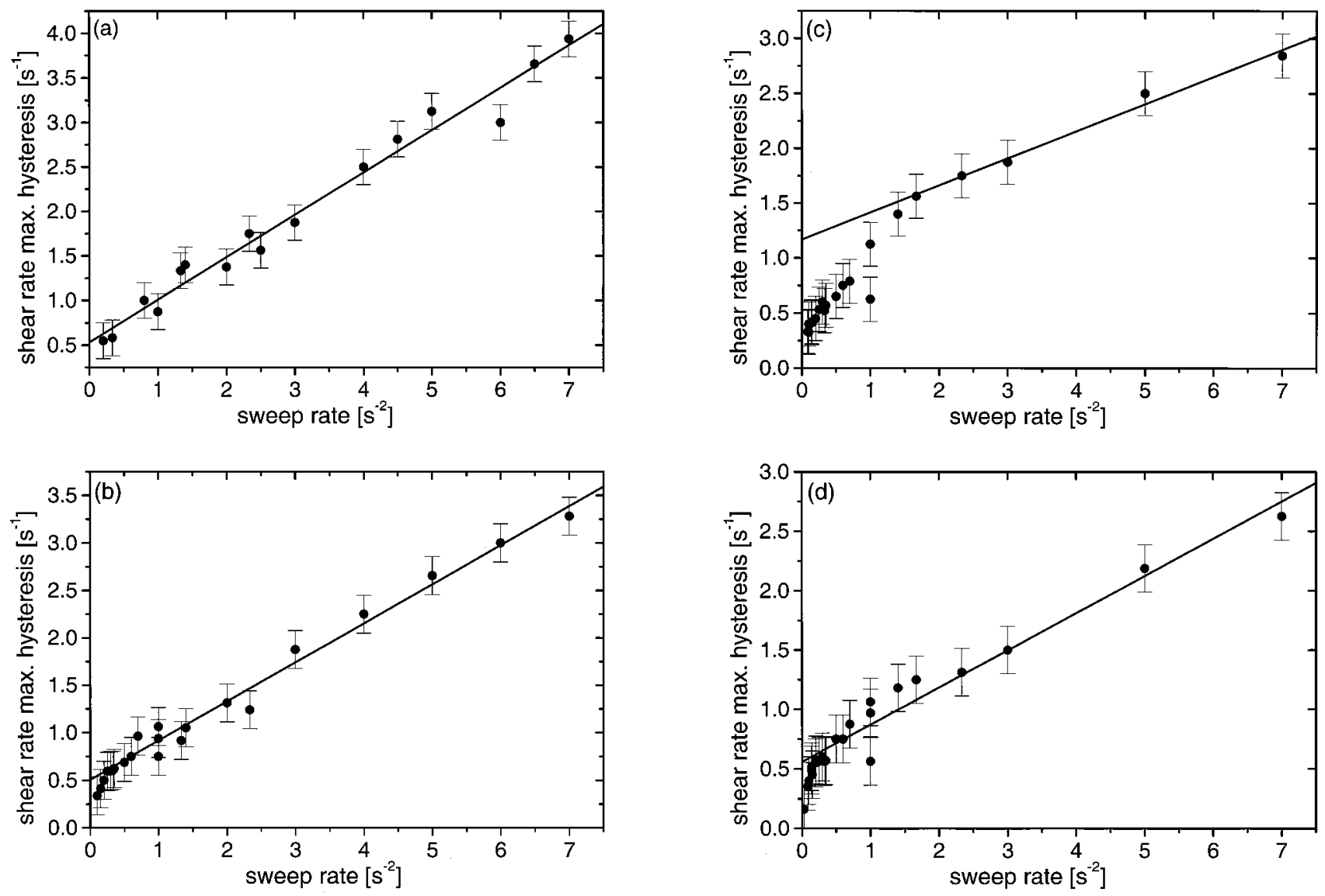

FIG. 11. The sweep rate dependence of the shear-rate where the maximum hysteresis is found, for the concentrations: (a) 5.84, (b) 10.59, (c) 11.18, and (d) $13.88 \mathrm{mg} / \mathrm{ml}$. The solid lines are linear fits, where data points for very small sweep rates are omitted.

sweep rate dependence of the shear-rate where the maximum in the hysteresis curves occurs, however, is experimentally found to be more sensitive on the sweep rate as compared to theory. The form of the hysteresis curves in Fig. 10(b) resembles the theoretical curves in Fig. 5(b), corresponding to the concentration regime II. The predicted gradual disappearance of a clear maximum in the regimes III and IV [see Figs. $5(\mathrm{c})$ and $5(\mathrm{~d})]$ is also observed in the experimental Fig. $10(\mathrm{c})$.

The sweep rate dependence of the shear-rate where the maximum hysteresis occurs is plotted in Fig. 11 for four different concentrations. For small sweep rates, the end shear rate is not large enough to actually cross the spinodal, so no hysteresis is expected. In the extrapolation procedure to zero sweep rate, we omitted data points for the very small sweep rates, where the sharp downward bend occurs. The shear-rate that is found from a linear extrapolation to zero sweep rate remains finite below the critical shear-rate. This "background" is independent of concentration and seems to be equal to a similar offset at very high concentrations. This discrepancy is not yet understood, and it cannot be explained by assuming that other kinds of structures (like lamellar structures) are formed, since the discrepancy is related to the low shear rate regime. The shear-rates found from linear extrapolations to zero sweep rates are plotted in Fig. 12.

The data in Fig. 12 should be compared to the dotted line in the nonequilibrium phase diagram in Fig. 3. The critical shear-rate that follows from Fig. 12 is $1.6 \mathrm{~s}^{-1}$. Comparing to the theoretically predicted critical Peclet number in Fig. 3 gives a bare rotational diffusion coefficient of $11 \mathrm{~s}^{-1}$. This value has been used in the previous subsection to compare experimental shear alignment curves with theoretical predictions.

\section{SUMMARY AND CONCLUSIONS}

Time resolved birefringence measurements have been used to probe the shear dependent location of isotropicnematic spinodals in a suspension of fd virus. The shear-rate

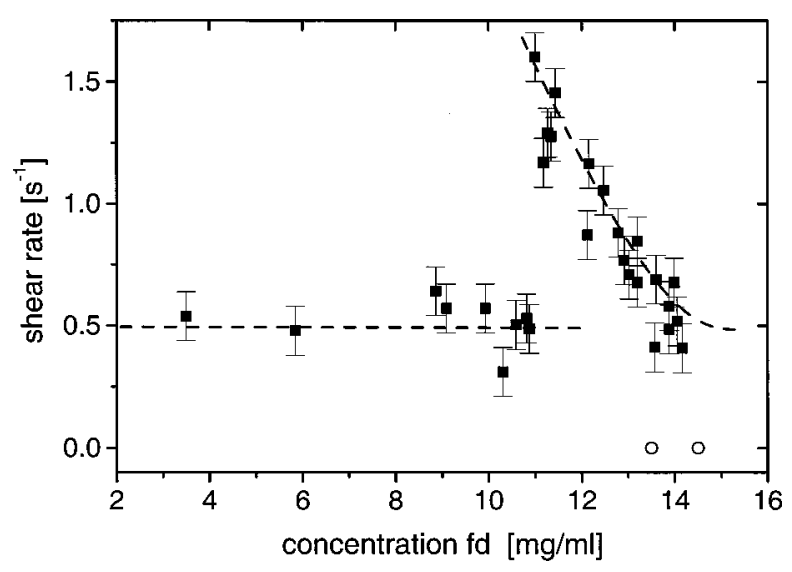

FIG. 12. The experimentally obtained nonequilibrium phase diagram. This plot corresponds (in part) to the dotted line in Fig. 3. The points $\bigcirc$ mark the binodal concentrations without shear flow. A dotted line is plotted to guide the eye. 
where the maximum hysteresis occurs during a shear sweep, where the shear-rate is first increased and subsequently decreased, lies in between the paranematic-to-nematic and the nematic-to-paranematic spinodals in the shear-rate vs concentration phase diagram. The critical shear-rate beyond which no distinction can be made between the nematic and paranematic states is found to be equal to $1.6 \mathrm{~s}^{-1}$. The corresponding critical concentration of fd virus is $10.9 \mathrm{mg} / \mathrm{ml}$, which should be compared to the binodal concentrations in the absence of shear flow of $13.0 \mathrm{mg} / \mathrm{ml}$ and $14.5 \mathrm{mg} / \mathrm{ml}$. There is thus an appreciable shear-induced shift of the spinodals, since the spinodal concentrations without shear flow are in between the binodal concentrations.

The birefringence hysteresis curves are calculated from an equation of motion for the orientational order parameter tensor, which is derived here from the $N$-particle Smoluchowski equation for rigid, lyotropic rods. This equation of motion is believed to be only qualitatively correct as a result of a Ginzburg-Landau type of expansion that is employed in its derivation, and due to the use of an approximate closure relation for a fourth order ensemble average. A very similar equation of motion has been considered by Doi and Edwards. ${ }^{19}$ There is one experimental observation which is not accounted for by the abovementioned equation of motion. There is a "background" shear-rate outside the spinodal region, that is, the shear-rate where the maximum hysteresis occurs at zero sweep rates is found to be nonzero. This discrepancy is not yet understood. In addition, shear alignment suspensions lose their birefringence on cessation of shear flow, where the equation of motion predicts a stable nematic state. This is probably due to the formation of inhomogeneities.

${ }^{1}$ H. Zocher, Anorg. Allg. Chem. 147, 91 (1925).

${ }^{2}$ J. D. Bernal and I. Fankuchen, J. Gen. Physiol. 25, 111 (1941).

${ }^{3}$ O. Pelletier, P. Davidson, C. Bourgaux, and J. Livage, Europhys. Lett. 48, 53 (1999).

${ }^{4}$ U. Kreibig and C. Wetter, Z. Naturforsch. 35, 750 (1980).

${ }^{5}$ A. J. Hurd, S. Fraden, F. Lonberg, and R. B. Meyer, J. Phys. (France) 46, 905 (1985).
${ }^{6}$ M. P. B. van Bruggen, J. K. G. Dhont, and H. N. W. Lekkerkerker, Macromolecules 32, 2256 (1999).

${ }^{7}$ X. M. Dong, T. Kimura, J. F. Revol, and D. G. Gray, Langmuir 12, 2076 (1996).

${ }^{8}$ T. Folda, H. Hoffmann, H. Chanzy, and P. Smith, Nature (London) 333, 55 (1988).

${ }^{9}$ M. Doi and S. F. Edwards, J. Chem. Soc., Faraday Trans. 2 74, 560 (1978).

${ }^{10}$ M. Doi and S. F. Edwards, J. Chem. Soc., Faraday Trans. 2 74, 918 (1978).

${ }^{11}$ S. F. Edwards and K. E. Evans, J. Chem. Soc., Faraday Trans. 2 78, 113 (1982).

${ }^{12}$ T. Sato and A. Teramoto, Macromolecules 24, 193 (1991).

${ }^{13}$ I. Teraoka and R. Hayakawa, J. Chem. Phys. 89, 6989 (1988).

${ }^{14}$ J. Tang and S. Fraden, Phys. Rev. Lett. 71, 3509 (1993).

${ }^{15}$ L. Onsager, Ann. N.Y. Acad. Sci. 51, 627 (1949).

${ }^{16}$ T. Odijk, Macromolecules 19, 2073 (1986)

${ }^{17}$ G. J. Vroege and H. N. W. Lekkerkerker, Rep. Prog. Phys. 55, 12141 (1992).

${ }^{18}$ P. van der Schoot, Technical University Delft, 1992.

${ }^{19}$ M. Doi and S. F. Edwards, The Theory of Polymer Dynamics (Clarendon, Oxford, 1986)

${ }^{20}$ T. Shimada, M. Doi, and K. Okano, J. Chem. Phys. 88, 7181 (1988).

${ }^{21}$ J. W. Winters, T. Odijk, and P. van der Schoot (preprint).

${ }^{22}$ P. Boltenhagen, Y. Hu, E. F. Matthys, and D. J. Pine, Phys. Rev. Lett. 79, 2359 (1997)

${ }^{23}$ P. D. Olmsted and P. M. Goldhart, Phys. Rev. A 46, 4966 (1992).

${ }^{24}$ P. D. Olmsted and C.-Y. D. Lu, Phys. Rev. E 60, 4397 (1999).

${ }^{25}$ P. D. Olmsted and C.-Y. D. Lu, Faraday Discuss. 112, 183 (1999).

${ }^{26}$ J. K. G. Dhont, Phys. Rev. Lett. 76, 4269 (1996).

${ }^{27}$ W. Hess, Rheol. Acta 23, 477 (1984).

${ }^{28}$ M. Doi, T. Shimada, and K. Okano, J. Chem. Phys. 88, 4070 (1988).

${ }^{29}$ J. K. G. Dhont, An Introduction to Dynamics of Colloids (Elsevier, New York, 1996).

${ }^{30}$ W. Maier and A. Saupe, Z. Naturforsch. A 13, 564 (1958).

${ }^{31}$ W. Maier and A. Saupe, Z. Naturforsch. A 14, 882 (1959).

${ }^{32}$ W. Maier and A. Saupe, Z. Naturforsch. A 15, 287 (1960).

${ }^{33}$ E. J. Hinch and L. G. Leal, J. Fluid Mech. 76, 4269 (1976).

${ }^{34}$ R. F. J. Kayser and H. J. Racheché, Phys. Rev. A 17, 2067 (1978).

${ }^{35}$ A. V. Bhave, R. K. Menon, R. C. Armstrong, and R. A. Brown, J. Rheol. 73, 413 (1993).

${ }^{36}$ G. G. Fuller, Optical Rheometry of Complex Fluids (Oxford University Press, New York, 1995).

${ }^{37}$ S. Fraden, Phase Transition in Colloidal Suspensions of Virus Particles (Kluwer Academic, Dordrecht, 1995).

${ }^{38}$ The hysteresis curves discussed in Sec. II F are obtained with a rotational diffusion coefficient equal to $20 \mathrm{~s}^{-1}$. The essential features of the hysteresis curves do not change when a value of $11 \mathrm{~s}^{-1}$ is used instead. 